This is the submitted version of the following article:

Soler M., Estevez M.-C., Moreno M.D.L., Cebolla A., Lechuga L. M. . Label-free SPR detection of gluten peptides in urine for non-invasive celiac disease follow-up. Biosensors and Bioelectronics, (2016). 79. : 158 -

10.1016/j.bios.2015.11.097,

which has been published in final form at https://dx.doi.org/10.1016/j.bios.2015.11.097 @ https://dx.doi.org/10.1016/j.bios.2015.11.097. This manuscript version is made available under the CC-BY-NC-ND 4.0 license http://creativecommons.org/licenses/by-nc-nd/4.0/ 


\section{Label-free SPR detection of gluten peptides in urine for non- invasive celiac disease follow-up}

Maria Soler ${ }^{\mathrm{a}, \mathrm{b}, 1}$, M. Carmen Estevez ${ }^{\mathrm{b}, \mathrm{a}, *}$, Maria de Lourdes Moreno ${ }^{\mathrm{c}}$, Angel Cebolla ${ }^{\mathrm{d}}$ and Laura M. Lechuga ${ }^{a, b}$

${ }^{a}$ Nanobiosensors and Bioanalytical Applications Group (NanoB2A), Institut Català de Nanociència i Nanotecnologia (ICN2), CSIC, 08193 Bellaterra (Barcelona), Spain

${ }^{\mathrm{b}}$ CIBER-BBN Networking Center on Bioengineering, Biomaterials and Nanomedicine, Spain

${ }^{c}$ Department of Microbiology and Parasitology, Faculty of Pharmacy, University of Seville,c/Profesor García González, S/N, 41012, Seville, Spain

${ }^{d}$ Biomedal S.L., c/Américo Vespucio 5, 41092 Seville, Spain

*mcarmen.estevez@cin2.es

${ }^{1}$ Present address: Ecole Polytechnique Federale de Lausanne, EPFL-STI-IBI-BIOS, CH-1015 Lausanne, Switzerland

*Corresponding Author:

M-Carmen Estevez

NanoBiosensors and Bioanalytical Applications Group

Institut Català de Nanociència i Nanotecnologia (ICN2)

Campus UAB - ICN2 Building

08193 Bellaterra (Barcelona), Spain

Tel: $\underline{+34937374629}$

email: mcarmen.estevez@cin2.es 


\begin{abstract}
Motivated by the necessity of new and efficient methods for dietary gluten control of celiac patients, we have developed a simple and highly sensitive SPR biosensor for the detection of gluten peptides in urine. The sensing methodology enables rapid and label-free quantification of the gluten immunogenic peptides (GIP) by using G12 mAb. The overall performance of the biosensor has been in-depth optimized and evaluated in terms of sensitivity, selectivity and reproducibility, reaching a limit of detection of $0.33 \mathrm{ng} \cdot \mathrm{mL}^{-1}$. Besides, the robustness and stability of the methodology permit the continuous use of the biosensor for more than 100 cycles with excellent repeatability. Special efforts have been focused on preventing and minimizing possible interferences coming from urine matrix enabling a direct analysis in this fluid without requiring extraction or purification procedures. Our SPR biosensor has proven to detect and identify gluten consumption by evaluating urine samples from healthy and celiac individuals with different dietary gluten conditions. This novel biosensor methodology represents a novel approach to quantify the digested gluten peptides in human urine with outstanding sensitivity in a rapid and non-invasive manner. Our technique should be considered as a promising opportunity to develop Point-of-Care (POC) devices for an efficient, simple and accurate gluten free diet (GFD) monitoring as well as therapy follow-up of celiac disease patients.
\end{abstract}

KEYWORDS: surface plasmon resonance; gluten immunogenic peptides; 33-mer gliadin peptide, gluten detection, celiac disease, $\mathrm{POC}$ device 


\section{INTRODUCTION}

Celiac disease (CD) is a chronic autoimmune disorder induced in genetically susceptible individuals by the ingestion of gluten proteins contained in wheat, barley, rye and certain varieties of oats. The disease is triggered by the presence of partially digested gluten peptides in the gastrointestinal tract, part of them are highly reactive to celiac $\mathrm{T}$ cells causing inflammation of the small intestine (Comino et al. 2011; Denham and Hill 2013). The only effective treatment for CD is a lifelong gluten-free diet (GFD). The strict adherence to a GFD is crucial to resolve symptoms and nutritional deficiencies, but also to avoid clinical complications associated with long-term gluten intake in celiac patients, such as osteoporosis, anemia or malignancy (Freeman 2012). It is generally recommended that individuals suffering CD have a careful therapy follow-up and dietary control. Extensive clinical guidelines have been reported about the importance of long-term monitoring of CD patients (Rubio-Tapia et al. 2013). However, the current markers for an efficient dietary control still remain unclear and the available methods involve risky and costly procedures (Silvester and Rashid 2007). Serological analysis of IgA antibodies involved in CD immunopathogenesis (e.g. tissue transglutaminase antibodies) have shown poor specificity and sensitivity for detecting either adherence to a GFD or intestinal damage recovery (Walker and Murray 2011). The use of serial endoscopies or biopsies is not useful neither since it is not considered ethical practice. Other suggested dietary controls, such as the fecal calprotectin test or intestinal permeability tests, can measure the consequences of gluten intake but they do not avoid the harmful aftermaths, they require expensive laboratory analysis and they are not concluding about the adherence to a GFD (Duerksen et al. 2005; Ertekin et al. 2010). Hence, a more direct and non-invasive control of gluten ingestion could trigger a significant progress towards the fabrication of the reliable Point-of-Care (POC) devices that would facilitate a continued follow-up of GFD compliance and therapy assessment.

Previous studies have highlighted the $\alpha$-gliadin 33-mer peptide as a valuable marker for gluten detection (Bethune et al. 2009). The gliadin 33-mer peptide is resistant to breakdown by gastrointestinal enzymes and persists in the gut, being the major cause of the immunotoxicity of wheat gluten. Antibodies against the dominant immunogenic peptide $\alpha$-gliadin 33-mer, A1 and G12 mAbs, have demonstrated to be practical tools to detect specifically the most active gluten immunogenic peptides (GIP) not only in food and beverages (Comino et al. 2013; Morón et al. 2008b; Real et al. 2014) but also in human stool after gluten intake (Comino et al. 2012). Comino et al. proved the feasibility of quantifying gluten peptides in feces employing the G12 mAb and evidenced its usefulness as indicator of gluten ingestion for dietary monitoring. However, feces analysis requires protein extraction and sample pretreatment to be done in laboratory infrastructures that restrict the final implementation in POC devices. 
The combination of more easy-to-handle samples such as urine and user-friendly biosensors could be very convenient for the development of portable and simple devices for the GFD compliance of celiac patients. Biosensors offer significant advantages over conventional techniques (e.g. mass spectrometry) enabling biochemical analysis with high sensitivity and excellent reproducibility in few minutes. Among them, optical biosensors and, especially, Surface Plasmon Resonance (SPR) biosensors play an outstanding role due to their ability to detect and quantify target compounds in real time without the use of labels or amplification steps. SPR biosensors exploit the high sensitivity of an evanescent field generated at the interface of a nanometric metal layer (typically Au layer of $\sim 50 \mathrm{~nm}$ thickness) and a dielectric when light strikes the interface under appropriate angle conditions. The properties of the evanescent field are highly dependent on the refractive index (RI) of the external media in such a way that small changes like those induced by biomolecular interactions (Homola 2008) can be measured. Biological recognition events occurring on the sensor surface can be monitored by tracking alterations in the characteristics of the reflected light, like the intensity or the resonance wavelength position. SPR biosensors have demonstrated their versatility as analytical platforms to monitor biomolecular interactions and to detect a wide range of analytes in a vast number of fields, including food safety, environmental monitoring or medical diagnostics and therapy control (Homola 2008).

By using specific antibodies against the peptide $\alpha$-gliadin 33-mer we have developed a novel methodology based on an indirect competitive immunoassay for the detection of most relevant GIP in urine employing a SPR biosensor. The optimization of the immunoassay has been directed towards the enhancement of the analytical features and especially to deal with possible interferences coming from the urine matrix. The rapid and quantitative detection of GIP in urine achieved with the SPR sensor exemplifies an attractive approach for the achievement of next-generation non-invasive and label-free analytical tools for the development of a POC device for dietary control of celiac patients.

\section{MATERIALS AND METHODS}

\subsection{Reagents}

Acetone, ethanol and hydrochloric acid were provided by Panreac (Barcelona, Spain). Alkanethiols for SAM formation (16-mercaptohexadecanoic acid (MHDA) and 11-mercaptoundecanol (MUOH), reagents for carboxylic groups activation (1-ethyl-3(3-dimethylaminopropyl) carbodiimide hydrochloride (EDC) and sulfo-N-hydroxysuccinimide (s-NHS), and Tween 20 were purchased from Sigma Aldrich (Steinhem, Germany). Copolymer poly-L-lysine grafted polyethylene glycol (PLL(20)$\mathrm{g}$ [3.5]-PEG(2)) was from Susos (Dübendorf, Switzerland). Monoclonal antibody G12, prolamin working group (PWG) gliadin and 33-mer gliadin peptide were from Biomedal S.L. (Seville, Spain).

\subsection{Study patients and urine sampling}


The local Ethics Committee of the Hospital de Valme (Seville, Spain) approved the study protocol. Written consent was obtained from adult patients and, in the case of children, from parents or legal guardians. Exclusion criteria for all study patients included the presence of known medical disease, use of prescription medications and antibiotics in the 2 months prior to the inclusion in the study. Moreover, healthy patients had no digestive disease symptoms or family history of CD.

All participants were provided of sterile containers. Urine specimens $(50-100 \mathrm{~mL})$ from volunteers were collected and stored at $-20^{\circ} \mathrm{C}$ until analysis. Urines from healthy subjects and celiac patients were collected under different gluten dietary conditions and times, as follows: (1) for the optimization and assessment, urines from celiac patients on strict GFD (> 2 years) were used, (2) to test the usefulness of the method, urines from healthy individuals on normal gluten containing diet were collected. All the samples were previously analyzed by using proprietary method of Biomedal (Seville, Spain) with a lateral flow test (Moreno et al. 2015; submitted).

\subsection{SPR biosensor description}

We employed a homemade SPR sensor based on the Kretschmann configuration, working at a fixed angle of incidence and monitoring the binding events in real time by tracking the SPR-wavelength displacements $\left(\Delta \lambda_{\text {SPR }}\right)$. Gold sensor chips (glass surface coated with $1 \mathrm{~nm}$ of Ti and $45 \mathrm{~nm}$ of $\mathrm{Au}$ ) were clamped between a trapezoidal glass prism contacting the sample through RI matching oil $(\mathrm{n} \approx 1.512)$ and a custom-made Delrin flow cell (volume $=4 \mu \mathrm{L}$ ), which is connected to a delivery microfluidic system consisting on a syringe pump with adjustable pumping speed that ensured a constant liquid flow and a manually operated injection valve. The sensor surface is excited by a collimated halogen light source set in TM polarization. The light reaches the substrate at a fixed angle of incidence $(\theta=$ $70^{\circ}$ ) through the prism coupling and the reflected light is collected and fiber-coupled to a CCD spectrometer (Jazz Module, Ocean Optics, USA). Tracking of the real-time resonance peak position is achieved via polynomial fit using custom-made readout software. The obtained sensorgrams show the displacements of the $\lambda_{\text {SPR }}$ to lower energy (higher RI, binding event) or higher energy (lower RI, unbinding event). Experiments were carried out keeping a constant flow rate of $25 \mu \mathrm{L} \cdot \mathrm{min}^{-1}$ for target recognition, and of $50 \mu \mathrm{L} \cdot \mathrm{min}^{-1}$ for regeneration. Total sample analysis was $20 \mathrm{~min}$ (40 min when including preincubation with the antibody and the regeneration step). Gold surface biofunctionalization is described in the Supporting Information.

\subsection{Competitive immunoassay performance}

To study the performance of the competitive immunoassay, PBST (PBS + 0.5\% Tween 20) was set as running buffer. The sample containing gluten peptides was preincubated for $15 \mathrm{~min}$ with a fixed concentration of antibody $\left(2 \mu \mathrm{g} \cdot \mathrm{mL}^{-1}\right)$ at room temperature (RT) in gentle agitation. The mixture was flowed over the biofunctionalized surface and binding event was monitored, obtaining signals 
inversely proportional to the concentration of the analyte in the sample. Regeneration of the PWG gliadin layer was carried out by injecting $5 \mathrm{mM} \mathrm{HCl}$ solution.

Calibration curves were obtained measuring different concentrations of gliadin 33-mer peptide by triplicate. Calibration curve fitting is described in the Supporting Information.

\section{RESULTS AND DISCUSSION}

\subsection{Design of the biosensor strategy}

By using the G12 mAb, we have designed a SPR-based indirect competitive immunoassay for the label-free detection of GIP, including the $\alpha$-gliadin 33mer peptide that is used as standard. In this format, the antigen or an analogous molecule is immobilized on the sensor surface and a fixed concentration of antibody is incubated with the sample containing the free target analyte. Then, the mixture is flowed onto the functionalized surface where free unbound antibodies (i.e. antibodies that have not interacted with the analyte in the sample) are captured by the immobilized antigen layer generating a signal inversely proportional to the analyte concentration in the sample. Competitive immunoassay was chosen over direct immunoassay (where the antibody is immobilized onto the sensor surface and the analyte is directly detected from the sample) because of the small size of excreted gluten peptides (MW $3.9 \mathrm{kDa}$ ). SPR detection is based on alterations of the RI of the dielectric caused by mass changes; thereby low molecular weight analytes $(<5 \mathrm{kDa})$ induce minute RI changes that can hinder the direct detection at low concentrations. Furthermore, competitive assays offer certain benefits in terms of robustness when compared to direct immunoassays. Generally, the immobilization of antibodies on solid supports diminishes their biological activity either by alteration of the antigen binding sites or due to the use of harsh conditions to regenerate the biosurface. In contrast, competitive immunoassay employs native or intact antibodies for the analyte recognition and the immobilization of the antigen usually provides a higher surface stability and allows the reutilization of the device for a higher number of measurements. However, it is also especially important in indirect competitive immunoassays the selection of a proper coating antigen, which, in combination with the specific antibody, is going to define the sensitivity and specificity of the final assay. In our work, both the $\alpha$-gliadin 33-mer peptide and the whole gliadin protein are good candidates for the development of the competitive assay. The 33-mer peptide comes precisely from the gluten hydrolysis and both have been previously used in the development of competitive ELISA using the same specific antibody (Morón et al. 2008a; Morón et al. 2008b).

Whereas for conventional ELISA the simple adsorption of the antigens on the solid support is optimal and commonly the preferred option, in the case of biosensors this step becomes more critical and the achievement of optimal performance strongly depends on the surface functionalization. The strategy for gold chip modification and the grafting density of the antigen are crucial factors to enhance the 
efficiency of the immunoassay and also to modulate and eventually minimize undesired nonspecific effects when real samples are evaluated. SPR gold chips are commonly functionalized taking advantage of the thiol-gold chemistry, which provides a strong attachment of thiol-modified molecules to gold surfaces by chemisorption. We employed long-chain alkanethiol compounds (mercaptohexadecanoic acid, MHDA, and mercaptoundecanol, $\mathrm{MUOH}$ ) in order to create a tight and uniform self-assembled monolayer (SAM) onto the gold substrate. Carboxylic acid groups of the SAM are used for covalent coupling of biomolecules by means of a carbodiimide-mediated linkage to exposed amine groups, usually from terminal lysine $(\mathrm{K})$ residues, establishing an amide bond. This strategy assures the high stability of the biofunctionalized surface and enables the control of the grafting density of antigens by varying the ratio of carboxylic/hydroxyl groups (MHDA:MUOH). As the gliadin 33-mer peptide does not carry any $\mathrm{K}$ residue within its amino acid sequence (LQLQPFPQPQLPYPQPQLPYPQPQLPYPQPQPF), we selected the protein (gliadin) as coating antigen (competitor in the indirect immunoassay). In particular we used the prolamin working group PWG gliadin (MW 33-45 kDa), which is considered an international reference reagent in gluten analysis due to the high content of gliadins, good solubility, homogeneity and stability (Van Eckert et al. 2006). A schematic representation of the SPR biosensing strategy is depicted in Figure 1.

\subsection{Optimization of the 33-mer gliadin peptide competitive immunoassay}

An in-depth optimization of the overall biosensing strategy was carried out to maximize the analytical features of the assay, in terms of sensitivity, selectivity and reproducibility. We evaluated several parameters, such as the surface biofunctionalization (SAM composition and antigen concentration), the fixed antibody concentration and the preincubation time. For the optimization study we employed a synthetic gliadin 33-mer peptide as target analyte. A detailed discussion of the optimization process and the results obtained are shown in the Supporting Information (SI). First, we tested different carboxylic/hydroxyl SAM ratios for PWG gliadin immobilization by comparing the efficiency to detect the G12 mAb being the ratio of $\mathrm{CO}_{2} \mathrm{H}: \mathrm{OH}$ of 1:1 the optimum one (see Figure S1.A in the SI). Immobilized gliadin and antibody concentrations were also selected according to the results obtained from titration experiments (noncompetitive recognition curves) as summarized in Figures S1.B and S1.C in the SI). We selected a concentration of PWG gliadin of $50 \mu \mathrm{g} \cdot \mathrm{mL}^{-1}$ and an antibody concentration of $2 \mu \mathrm{g} \cdot \mathrm{mL}^{-1}$. A preincubation step of 15 minutes led to complete signal inhibition (data not shown). This period was enough to ensure the formation of the immunocomplex and was fixed for further experiments. Regeneration of the PWG gliadin layer was also achieved under acid conditions $(5 \mathrm{mM} \mathrm{HCl})$ (Figure S1.E in the SI) being possible more than 100 cycles with good repeatability.

Additionally, we assessed the specificity of the assay by evaluating either a non-target protein which could also be found in the urine, the human Chorionic Gonadotropin, hCG, and a nonspecific antibody 
(control antibody, anti-C-reactive protein) (Figure S1.D in the SI). On one hand, incubation of G12 $\mathrm{mAb}$ with hCG at high concentration $\left(2 \mu \mathrm{g} \cdot \mathrm{mL}^{-1}\right)$ led to no inhibition, (i.e. maximum signal was obtained as in the case of zero concentration of analyte); on the other hand, the use of a control antibody resulted in negligible adsorption onto the surface indicating that signal corresponds exclusively to specific interaction of G12 mAb with the gliadin surface. Although the recognition pattern and cross-reactivity studies of the antibody against closely related gluten peptides has been previously performed (Morón et al. 2008a), these results corroborate not only the inherent specificity of the antibody but specially the selectivity of the antigen-coated layer to exclusively interact with the G12 mAb.

With all above selected conditions a calibration curve for the gliadin 33-mer peptide was obtained (Figure S1.F in the SI and Figure 2). An $\mathrm{IC}_{50}$ value of $5.64 \mathrm{ng} \cdot \mathrm{mL}^{-1}$ was achieved. The limit of detection $(\mathrm{LoD})$ and quantification $(\mathrm{LoQ})$ were 0.33 and $1.12 \mathrm{ng} \cdot \mathrm{mL}^{-1}$ respectively, and the linear working range was found between $1.12-19.20 \mathrm{ng} \cdot \mathrm{mL}^{-1}$. The SPR-based immunoassay showed comparable features to the ones obtained using the same reagents in different ELISA configurations $\left(\mathrm{LoD}<1 \mathrm{ng} \cdot \mathrm{mL}^{-1}\right.$ ) (Morón et al. 2008b). For evaluation of the reproducibility both intra- and interassay Coefficients of Variability (CV) of the main analytical parameters were calculated (Table 1). The mean values for the intra- and inter-assay $\mathrm{CV}$ were well-below the maximum variability recommended for clinical analysis ( 15\%) (Buick et al. 1990), confirming the excellent reproducibility and stability of the proposed immunoassay.

\subsection{Analysis of 33-mer gliadin peptide in urine matrix}

The urine is one of most attractive biological fluids for dietary control analysis due to the simple and non-invasive sample collection. Moreover, it can be obtained in large amounts. However, there are several limitations for the urinary analysis mainly related to low concentration of proteins, the high levels of salts or other interfering compounds and, more importantly, the high degree of variability. Parameters like $\mathrm{pH}$, osmolality, gravity or the concentration of certain components vary over a wide range between different specimens, the diet or the collection time (Sviridov and Hortin 2009). This large variability of urine samples represents an important barrier for the reliable detection and quantification of clinical biomarkers. To our knowledge, so far scarce studies have been reported for the label-free detection of small peptides in urine. Current analytical methods are usually based in mass-spectrometry techniques which are expensive and time-consuming, and require laborious pretreatment and extraction processes (Decramer et al. 2008). Hence, SPR-based biosensing of peptides in urine in general would have a great potential for fast and efficient clinical assays as well as for biomarker discovery. Nevertheless, the influence of matrix interferences and variability needs to be deeply optimized to provide accurate and reliable results. 
The feasibility of direct detection of gliadin 33-mer peptide in urine employing the biosensor was evaluated by studying the influence of the matrix components present in the fluid in the competitive assay performance. Although protein concentration is relatively low compared to other biological fluids like serum or plasma, normal healthy urine contents a total protein concentration between 50 $100 \mu \mathrm{g} \cdot \mathrm{mL}^{-1}$, and albumin can represent up to $20 \mu \mathrm{g} \cdot \mathrm{mL}^{-1}$ (Brunzel 2013). In SPR biosensors, where the signal comes indirectly from mass changes onto the sensor surface, the adsorption of proteins may lead to undesired nonspecific signals that must be minimized. In previous work, we evidenced the extraordinary resistance to protein fouling provided the copolymer poly-L-lysine grafted polyethylene glycol (PLL-g-PEG) as blocking additive (Soler et al. 2014; Soler et al. 2015). Therefore a blocking step with PLL-g-PEG $\left(0.5 \mathrm{mg} \cdot \mathrm{mL}^{-1}\right)$ after PWG gliadin immobilization was carried out to prevent nonspecific adsorptions of urine components. Moreover, running buffer was changed to PBST $0.5 \%$ (PBS with $0.5 \%$ of Tween 20, whose addition in the media has demonstrated to also help reduce the nonspecific fouling onto the surface (Soler et al. 2014)). The background signal observed under these conditions was low enough and highly reproducible (standard deviation below $0.01 \mathrm{~nm}$ ) to permit reliable detection of the peptides in urine (data not shown), thereby assuming a constant background for all measurements. On the other hand, the presence of proteins, the high salt concentration or the $\mathrm{pH}$ value may also affect the interaction between the antibody and the biofunctionalized surface, leading to important variations of the immunoassay behavior (i.e. alteration in the maximum signal, the slope and the detectability). The G12 mAb was diluted in different gluten-free urine samples collected from several individuals following strict GFD and evaluated with the SPR biosensor (data not shown). A significant variability of the signal (maximum signal at zero concentration of analyte) was obtained $(\mathrm{CV}=23.08 \%)$, which is attributed to the composition variability of the urine samples. In order to stabilize the behavior of the assay, urine samples were buffered by diluting them with PBST $0.5 \%$ (1:1). The variability (CV) was reduced to $2.6 \%$, improving the reproducibility and increasing the reliability of the measurements.

A calibration curve in urine diluted 1:1 with PBST 0.5\% was carried out using gliadin 33-mer as target analyte. Figure 2 shows and compares curves obtained for spiked GF urine and PBS buffer. Matrix constituents of urine did not produce significant interferences in the competitive immunoassay, leading to nearly identical analytical sensitivity than the one obtained in standard buffer conditions. The $\mathrm{IC}_{50}$ was determined at $5.06 \mathrm{ng} \cdot \mathrm{mL}^{-1}$, reaching a $\mathrm{LoD}$ of $0.46 \mathrm{ng} \cdot \mathrm{mL}^{-1}$ and a dynamic range between $1.20-21.55 \mathrm{ng} \cdot \mathrm{mL}^{-1}$. The sensitivity values achieved are comparable to those obtained with conventional ELISA, which are several orders of magnitude below the maximum recommended gluten concentration in the digestive tract (i.e. $<20 \mu \mathrm{g} \cdot \mathrm{mL}^{-1}$ ) (Catassi et al. 2007; Morón et al. 2008b). Moreover, our SPR-based assay permits rapid and label-free analysis of urine in a user-friendly performance without requiring any sample pretreatment which commonly results in 100-fold sample dilutions prior to the analysis. 


\subsection{Determination of gluten toxic peptides patient samples}

To evaluate whether our proposed SPR biosensing methodology was suitable to detect gluten toxic peptides in celiac patients, the analysis of real urine samples was attempted. So far, the experiments have been performed using the gliadin 33-mer peptide as standard analyte. As detailed before, the G12 mAb was produced against the gliadin 33-mer peptide from wheat gluten. However cross-reactivity experiments demonstrated the ability of the antibody of recognizing other immunogenic peptides from wheat and other toxic cereals for celiac, such as those from barley or rye (Morón et al. 2008a), with different degree of recognition and affinity. Therefore, the presence in urine of immunogenic peptides with different sequences from different cereal sources (equivalent peptides (33EPs)) would be expected, that will eventually react with the antibody and hence would be quantified. Besides, the gliadin 33-mer peptide is involved in numerous metabolic processes that could alter its structure or amino acid sequence, such as the transglutaminase-mediated deamidation (Morón et al. 2008a; Shan et al. 2002). These factors can influence on the immunoassay behavior, affecting the sensitivity of the analysis. Therefore, in order to have a more realistic standard analyte, we performed the biosensorbased immunoassay using as analyte the peptide mixture obtained from a urine sample from a healthy individual following a normal gluten-containing diet. The concentration of 33EPs in the sample was determined employing lateral-flow chromatographic strips and specific strip reader (Martin et al. 2014). In addition, part of this urine sample was subjected to an extraction and purification procedure obtaining a 33EP extract in PBS buffer. The purification procedure was performed using Biomedal SL proprietary technology. Both samples (untreated urine and PBS extract) contained a mixture of peptides at a concentration of $[33 \mathrm{EPs}]=20 \mathrm{ng} \cdot \mathrm{mL}^{-1}$. The samples were pre-concentrated and then serially diluted either in buffer or in GF urine, respectively, in order to cover the concentration range necessary to define the assay. Competitive assays were performed applying the optimized conditions described before. Figure 3 shows the calibration curves (green and blue lines) which were compared to the ones previously obtained using 33-mer gliadin peptide as pure standard (black and orange lines).

As can be observed, the detectability of the immunoassay was slightly worse (i.e. curves are shifted to higher analyte concentration range), leading to higher limits of detection (Table 2). The IC50 determined for 33EP detection in urine was $18.58 \mathrm{ng} \cdot \mathrm{mL}^{-1}$ and the $\mathrm{LoD}$ was $1.72 \mathrm{ng} \cdot \mathrm{mL}^{-1}$. These results can be attributed either to the presence of several peptide variants from different cereals, all of them with different affinity degree for the antibody and also to the probable presence of peptides containing structural modifications occurring during the metabolic processes. Both aspects eventually affect the resultant averaged sensitivity. However, it must be highlighted the high degree of similarity observed in the calibration curves performed in PBS and urine for the detection of 33EP in real samples (blue and green curves). These results obtained with a real mixture of gluten peptides confirm our previous results and demonstrates the viability of measuring directly in urine without the need of performing any kind of purification or extraction treatment. 
Finally, we attempted a preliminary qualitative evaluation of real samples. We measured several urine samples containing different concentration of gluten in order to test the usefulness of the SPR biosensor to identify gluten ingestion. Urine samples were collected from celiac patients from the Hospital de Valme (Seville, Spain) and healthy volunteer individuals with different diet conditions: (i) volunteers under a gluten-free diet with no transgression, (ii) low consumption of gluten of celiac patients under GFD but with signs of transgressions (weak positive in the lateral flow test and/or incomplete gut mucosa healing) and (iii) moderate/normal consumption of gluten (normal gluten containing diet of 5-30g/day with quantifiable levels of 33EP in the lateral flow test and/or celiac patients with high damage in intestinal mucosa). Four samples of each group were measured. Each sample was only diluted 1:1 with PBST and incubated with G12 mAb for $15 \mathrm{~min}$. The samples were then flowed over the functionalized sensor surface and measured in real time. Signals were interpolated in the calibration curve (Figure 3, green curve). Figure 4 compares the results obtained for each diet condition based on the determined statistical median of the interpolated concentration. Concentrations for every sample qualitatively correlate with the expected amount of gluten present in the urine. Besides, it can be observed a significant statistical difference between the individual populations. This result demonstrates that our SPR biosensor can be employed for monitoring the consumption of gluten by simple urine analysis and therefore opens up a way for the development of fast and easy-to-use POC devices for CD therapy follow-up.

\section{CONCLUSIONS}

We have developed a novel SPR biosensing methodology for simple and label-free detection of gluten toxic peptides in the urine of celiac patients. The strategy consists of a competitive immunoassay that employs a specific monoclonal antibody (mAb G12) with high affinity for gliadin 33-mer equivalent peptides (33EPs) coming from gluten proteins of wheat, barley and rye. The SPR biosensor has demonstrated to enable direct quantification of the small digestive peptides in urine without requiring previous extraction or purification procedures, performing the complete assay cycle in $20 \mathrm{~min}$. Several parameters have been optimized in order to obtain reproducible, selective and sensitive analysis, achieving a LoD of $1.72 \mathrm{ng} \mathrm{mL}^{-1}$ in real urine samples. This value is well-below the maximum recommended gluten concentration in the digestive tract for $\mathrm{CD}$ patients (i.e. $<20 \mu \mathrm{g} \mathrm{mL}^{-1}$ ) (Morón et al. 2008b). The biosensing approach has also demonstrated the feasibility of clearly identifying gluten consumption by measuring several urine samples from both healthy (normal diet) and celiac subjects (gluten-free diet). The preliminary qualitative assay has shown significant statistical differences between individuals with different diet conditions (GFD, low gluten consumption, normal diet) as well as good correlation between the calculated concentration and the expected amount of gluten peptides in the urine. This SPR analytical methodology constitutes a first approach towards the achievement of 
POC biosensors that permit rapid, accurate and non-invasive dietary control for the celiac disease follow-up.

\section{AKNOWLEDGEMENTS}

The authors want to thank Dr. Sergio Valenzuela and his family for kindly providing gluten-free urine samples for the study. M.S. acknowledges financial support from "Formación de Personal Investigador (FPI)" Program from the Spanish Ministry of Economy and Competitiveness (MINECO). The NanoB2A is a consolidated research group (Grup de Recerca) of the Generalitat de Catalunya and has support from the Departament d'Universitats, Recerca i Societat de la Informació de la Generalitat de Catalunya (2014 SGR 624). ICN2 is the recipient of Grant SEV-2013-0295 from the "Severo Ochoa Centers of Excellence" Program of Spanish MINECO.

Supporting Information. Supplementary data associated with this article (Gold surface biofunctionalization, calibration curve fitting and immunoassay optimization) can be found in the online version. This material is available free of charge via the Internet at http://www.sciencedirect.com

\section{REFERENCES}

Bethune, M.T., et al., 2009. J Biol Chem 16(8), 868-881.

Brunzel, N.A., 2013. Elsevier Health Sciences.

Buick, A., et al., 1990. J Pharm Biomed Anal 8(8), 629-637.

Catassi, C., et al., 2007. Am J Clin Nutr 85(1), 160-166.

Comino, I., et al., 2011. Gut, 1468-3288.

Comino, I., et al., 2013. J Sci Food Agric 93(4), 933-943.

Comino, I., et al., 2012. Am J Clin Nutr 95(3), 670-677.

Decramer, S., et al., 2008. Mol Cell Proteomics 7(10), 1850-1862.

Denham, J., Hill, I., 2013. Curr Allergy Asthma Rep 13(4), 347-353.

Duerksen, D.R., et al., 2005. Dig Dis Sci 50(4), 785-790.

Ertekin, V., et al., 2010. J Clin Gastroenterol 44(8), 544-546.

Freeman, H.J., 2012. Maturitas 73(3), 206-211.

Homola, J., 2008. Chem Rev 108(2), 462-493.

Martin, C.S., et al., 2014. Google Patents.

Morón, B., et al., 2008a. PLoS ONE 3(5), e2294.

Morón, B., et al., 2008b. Am J Clin Nutr 87(2), 405-414.

Real, A., et al., 2014. PLoS ONE 9(6), e100917.

Rubio-Tapia, A., et al., 2013. Am J Gastroenterol 108(5), 656-676.

Shan, L., et al., 2002. Science 297(5590), 2275-2279.

Silvester, J., Rashid, M., 2007. Can J Gastroenterol Hepatol 21(9), 557 - 564.

Soler, M., et al., 2014. Sensors 14(2), 2239-2258.

Soler, M., et al., 2015. Biosens Bioelec 66, 115-123.

Sviridov, D., Hortin, G.L., 2009. Clin Chim Acta 404(2), 140-143.

Van Eckert, R., et al., 2006. J Cereal Sci 43(3), 331-341. 
Walker, M.M., Murray, J.A., 2011. Histopathology 59(2), 166-179. 


\section{FIGURE LEGENDS}

Figure 1. Scheme representing the three steps in the development of the SPR-based competitive immunoassay: formation of a mixed alkanethiol SAM; PWG gliadin immobilization via covalent coupling through the amine terminal groups; immunoassay where a competition between PWG gliadin and gluten immunogenic peptides (GIP) for the free antibody takes place.

Figure 2. Calibration curves for the gliadin 33-mer peptide immunoassay in PBS buffer (black) and urine diluted 1:1 with PBST (orange). Each point represents the mean \pm SD of three replicates. Table compares main analytical parameters determined for both curves.

Figure 3. Calibration curves for competitive immunoassay of: (i) gliadin 33-mer peptide diluted in PBS (black); (ii) gliadin 33-mer peptide spiked in gluten-free urine (orange); (iii) Mixture of peptides (33EPs) extracted from purified positive urine diluted in PBS (blue) and (iv) Mixture of peptides (33EPs) from untreated positive urine diluted in gluten-free urine (green).

Figure 4. Analysis of patient's urine samples from individuals following a (i) gluten-free diet $(n=4)$, (ii) low gluten consumption diet $(\mathrm{n}=4)$ and (iii) normal diet with high/moderate consumption of gluten $(n=4)$. Median, maximum and minimum values are shown. 